\title{
THE SURGERY OF PULMONARY TUBERCULOSIS.
}

\author{
By H. MORRISTON DAVIES, M.A., M.D., M.Ch. (Cantab.), F.R.C.S. (Eng.).
}

(Medical Superintendent, Vale of Clwyd Sanatorium; Consulting Surgeon to University College Hospital, City of London Hospital for Diseases of the Heart and Lungs, King Edward VII. Welsh National Memorial Association, Lancashire County Sanatoriums, Cheshire Joint Sanatorium.)

\section{Introductory.}

It has been obvious for long enough that sanatorium treatment is limited in its effectiveness to secure healing for many cases of pulmonary tuberculosis. There are certain types which, owing to the nature of the mechanical and pathological changes, must be and have been proved, times without number, to be incapable of responding adequately and completely to simple bed-rest and fresh air or to drugs or injections. Those who in more recent years have practised or seen practised surgical intervention in many of these cases of pulmonary tuberculosis, or who have read some of the, by now, immense literature on the subject, know that surgery has been fully justified in its alliance with sanatorium treatment.

The value of surgery is not as a substitute or alternative for medicine: as a general rule surgery steps in only at that point when the limitations of medicine have been reached. Its use is then so to alter and adjust the mechanical disabilities which have developed that sanatorium treatment can again resume its beneficent work with the hope of a successful issue, previously impossible.

It is true that surgery is no longer confined to these limitations: the successes obtained have been too great. There are many cases which have not developed those mechanical changes which stultify sanatorium treatment and which might reasonably be expected to yield to this régime alone provided time is no object. but for which surgical measures are of value because they accelerate healing or ensure greater certainty or security. In respect of such cases, however, there is a very great difference between the comparatively simple methods of artificial pneumothorax and of phrenicectomy and the more serious operations of apicolysis and thoracoplasty. These latter are only used when other means of arresting the disease have failed and it becomes necessary to take more serious risks if life is to be saved.

\section{General Principles governing Surgical Intervention.}

The general principles of all forms of surgical treatment are to allow of reduction of the volume of the lung by lowering the intrapleural tension, and to obtain collapse of diseased areas and especially the complete approximation of the walls of cavities. Additional benefits are obtained by this relaxation of intrapleural and intrapulmonary tension, which enables the fibrous tissue to contract, not only harmlessly but beneficially, and enables cavities to close. They are the removal of the dangers of hæmorrhage, of cough metastases, of retention of sputum and of toxæmia, and, moreover, the immobilization of the diseased organ to a degree impossible by the most complete bed rest. 


\section{Cases suitable for Surgical Treatment.}

The cases which are suitable for some form or another of collapse therapy are to be found among those patients with acute pneumonic exudative or atelectatic tuberculosis, or with subacute disease of the exudative type, and especially those which are caseating and in which there is breaking down of lung tissue and cavity formation. They are to be found also among cases with proliferating fibrotic changes, particularly those with well established mechanical changes such as rigid wall cavities or multiple cavities. They are to be found, in addition, among patients with troublesome, persistent or dangerous symptoms, as for instance persistent irritating diaphragmatic cough, retention of sputum and toxæmia and hæmorrhage.

\section{Artificial Pneumothorax.}

Of the various methods of producing lung collapse, that by artificial pneumothorax is the simplest and the most universal. In an ideal case the collapse of the lung is practically complete and the control of the disease and of the symptoms therefrom is absolute. Unfortunately the ideal case is not frequent. In some cases universal adherence of the pleural membranes frustrates every endeavour. In the majority of cases adhesions between the membranes interfere to a greater or less extent with satisfactory collapse. At one end of the scale the extent of possible collapse is so insignificant that the method must be abandoned: at the other end, the few adhesions stretch and are of no serious consequence. Between these extremes there is every degree of possible success or failure. A partial collapse which produces relaxation over and partial control of the diseased area is worth maintaining: one which collapses the greater part of the lung but leaves unaffected a large apical cavity is probably valueless. When adhesions are few and of sufficient length to allow of access to them, they may be capable of division and so enable a partial and unsuccessful artificial pneumothorax to be converted into a complete and effective one.

Adhesions are due to inflammatory changes close to the surface of the lung implicating the pleura: they are therefore found most frequently over the diseased area and tend to interfere with just that portion of the lung it is most necessary to collapse. When there are adhesions in the neighbourhood of a cavity whilst the rest of the lung cavity is comparatively free, the cavity becomes "suspended" and then often increases in size (Fig. I, Plate I). Tension has been exerted on opposing walls of the cavity by the adhesions, thus producing the exact opposite to the desired relaxation. As a result of this it is not uncommon for a cavity in the upper lobe, which is scarcely recognizable before artificial pneumothorax is started, to become very well defined and obvious once the pneumothorax is established. Unless this unsatisfactory state of affairs can be overcome either by division of the adhesions or by a compensating upward relaxation of the lung produced by hemidiaphragmatic paralysis, the artificial pneumothorax will have to be abandoned.

Pleural effusions are the commonest complication of artificial pneumothorax. They are infinitely more common relatively when adhesions are present than when there is a complete and free collapse. Tuberculous disease reaching to the surface of an uncollapsed lung will cause an inflammatory change in the opposed pleural membranes which, in all probability, unless acute, seals off this area from the rest 
of the pleural cavity. This rarely happens when the lung is collapsed, as a lung fully collapsed has its disease under greater control than when there are adhesions, and, consequently, surface extension is less likely to occur. The adhesions themselves are, moreover, a source of danger. The drag on them, especially when there is a high intrapleural pressure, as during coughing, may irritate the lung surface, or actually cause rupture of finer strands, possibly thus liberating tubercle bacilli direct into the pleural cavity.

The onset of an effusion is usually indicated by a feeling of malaise, and a sharp rise of temperature which may persist for several days before it falls by lysis. As a general rule an effusion should not be interfered with. A small portion may be drawn off to ascertain the character of the fluid and to find out whether it is a simple serous one, a tuberculous infection or, rarely, a mixed infection.

The indications for aspiration of a non-pyogenic effusion are a persistent pyrexia due to the effusion, a progressive effusion which is producing an increasing intrapleural pressure, or a large effusion which will not absorb. During aspiration the desired intrapleural pressure and collapse of the lung must always be maintained by replacement by air or oxygen. When the effusion is due to a tuberculous infection, sterilization must be attempted at first by the injection of 5 c.c. of a 2 per cent. solution of methylene blue. If this does not succeed, the fluid must be aspirated and replaced by a 5 per cent. solution of olive oil-gomenol.

In some cases of acute tuberculization of the pleural cavity and in all those with a mixed infection the toxæmia is so intense and persistent that even repeated replacement by olive oil-gomenol fails to check it. It is necessary then to institute air-tight continuous drainage and irrigation of the cavity with Dakin's solution or normal saline. Contrary to the customary assertions, an air-tight drain can be removed once the pleural cavity has been rendered sterile, and closure of the wound obtained. On the other hand, an air-tight drain can be maintained for weeks if it is desired.

Spontaneous pneumothorax is a less common complication than effusion. The disturbance caused thereby varies from discomfort and possibly a simple serous effusion when the opening is a very small one, to intense distress and a pyogenic infection, and even death, in the serious cases. Most commonly there is pain, dyspnoea and acute discomfort from the rapid rise in intrapleural pressure. Excessive collapse and mediastinal displacement are usually checked either by the increased collapse obliterating the opening, or by adhesions steadying the lung and mediastinum.

In the less severe cases symptomatic treatment only is necessary. If the pressure is distressing and progressive, air will have to be drawn off. Care must, however, be taken not to reduce the intrapleural pressure to an extent likely to reopen the pleuro-pulmonary sinus if that is already closed by the increased lung collapse and possibly by lymph. Severe cases need immediate puncture to let the gas escape. When there is a valve opening in the lung and a progressive pneumothorax, an air-tight drain must be passed through the chest wall:

Except for the operation of phrenicectomy which is sometimes done as an alternative, artificial pneumothorax is the method of choice in almost all cases when collapse therapy is indicated. It is the only method in acute and subacute cases. Lung collapse by artificial pneumothorax can be carried out even when 
there is bilateral disease, provided always that the disease in the contralateral lung is not too acute nor too extensive. An associated laryngeal involvement is an indication rather than a contraindication. Even a mild degree of intestinal tuberculosis does not preclude collapse therapy by artificial pneumothorax.

\section{Bilateral Disease.}

The treatment of bilateral disease by bilateral operative treatment is frequently done nowadays. The most usual methods are an artificial pneumothorax on one side and a phrenicectomy on the other, or a bilateral artificial pneumothorax. A bilateral artificial pneumothorax is occasionally started simultaneously on the two sides, but it is much easier and safer to get one lung under control before attempting the contralateral side. A partial collapse only of both sides is possible and all unnecessary strain on the heart must be avoided. Other possible combinations are an artificial pneumothorax on the one side and an oleothorax on the other (Fig. 2, Plate I). An artificial pneumothorax of the one lung and an apicolysis or even an apical thoracoplasty of the other may on occasion be permissible. The author has a patient in whom the apex of the right lung, which contained a thickwalled cavity, is collapsed by an apical thoracoplasty: this is combined with an oleothorax on the same side controlling the middle and lower lobes and a selective collapse of the contralateral lung.

\section{Oleothorax.}

The value of gomenol in oil in the treatment of effusions has already been referred to. Paraffin and 2 per cent. gomenol can also be used to replace an artificial pneumothorax when, for any reason such as difficulty in arranging for refills, it is impracticable to maintain the lung collapse by air. The advantage of it is the very slow rate of absorption. Paraffin gomenol must be used with the very greatest caution. It must not be introduced into the pleural cavity until the membranes have shewn prolonged tolerance to air. Until tolerance to the paraffin gomenol is acquired the amount run in must be very small. The maximum initial dose is 2 c.c. : even this may produce a severe reaction. Subsequent doses may be double the previous one unless the reaction is excessive.

Paraffin and gomenol must never be allowed to fill completely a pleural space bounded by rigid walls. The contraction of the walls onto the paraffin will raise the pressure progressively and there is then great danger of ulceration of the surface of the lung and of the formation of a pleuro-pulmonary fistula. If the walls are rigid, an air pocket must be maintained above it or the oil removed. When, however, the mediastinum is mobile it is safe to fill the pleural cavity with the oil.

\section{Phrenic Evulsion and Phrenicectomy.}

Hemidiaphragmatic paralysis is produced either by evulsing the phrenic nerve whereby the continuity of the nerve itself and of all accessory branches is broken, or by phrenicectomy: the latter implies the excision, of a length of the main phrenic trunk, of any accessory nerve bundles and of the nerve to subclavius. When a temporary effect only is desired, the main phrenic nerve is crushed and the other sources of supply are divided. Phrenic evulsion is not as safe or certain a method as phrenicectomy. If the nerve is bound down by thickened pleura in its intrathoracic course, the drag on the nerve may tear the pleura and even the lung, and cause a pneumothorax, or expose the mediastinum 
to infection. Veins also, which have become adherent to the nerve, have been torn, with, on rare occasions, disastrous result. Whilst these accidents are rare, it is quite common for the nerve to break off short during the attempt at evulsion, leaving the distal part of the nerve still in uninterrupted continuity with its cervical root origin by way of accessory nerve bundles.

The effect of destroying the phrenic nerve fibres is to paralyse the dome of the diaphragm so that not only is it neither in a state of tonic contraction, nor does it descend with inspiration, but during a forceful inspiratory act it will actually rise into the thorax: that is to say its movements are paradoxical. The other result of the paralysis and loss of tone is that the dome assumes a higher position in the thorax and one that tends to increase during the ensuing months. The extent of this rise varies, however, very greatly in different individuals-it may be $2 \mathrm{~cm}$. only or it may be as much as $10 \mathrm{~cm}$.- depending on the condition of the pleura, whether thickened or adherent, and the character of the changes in the lung, whether densely fibrosed and shrunken or the seat of thick wall cavities or of caseating exudative disease.

These two results of phrenicectomy each have their separate value. The rise of the dome is of major importance when the object of the operation is the relaxation of the fibrous tissue changes in the lung: in other words when the operation is done as a method of collapse therapy. The loss of tone and the paralysis are the important changes when the aim has been to relieve symptoms such as pain and diaphragmatic cough.

The influence of the rise of the dome extends throughout the lung. The characteristic of fibrous tissue is that it is always shrinking and exerting a contracting force on the affected part of the lung. This action is, however, opposed by the rigidity of the surrounding walls and the opposing, expanding pull of the inspiratory act. When there are considerable but localised fibrotic changes, the reduction in the hemithorax enables this fibrous tissue to contract and to reduce the volume of the affected part. In a case therefore of chronic upper lobe or apical disease, this part of the lung may be able to take greater advantage of the reduction in space than the healthy lower lobe: the collapse is then selective in character. This selective action is however dependent on the absence of interlobar and interpleural thickening and adhesions which may prevent the effect of the raised, paralysed dome being transmitted to the upper lobe. If the disease is distributed fairly uniformly throughout the lung, then the influence of the paralysed, raised dome will be greater on the lower part of the lung. (Fig. 3, Plate I.)

The indications for hemidiaphragmatic paralysis are numerous. It can be stated as a general rule that a phrenicectomy should be done on every case in which an artificial pneumothorax has been tried and has been found inadequate or impracticable. The only exception to this is for those patients for whom a major operation, such as complete thoracoplasty, is the alternative. It must, however, be realized that if one of the objects of the artificial pneumothorax is the obliteration of a large thick-walled cavity, the phrenicectomy is most unlikely to produce more than a slight diminution in its size.

Phrenicectomy is used extensively in combination with artificial pneumothorax. It is of value in all cases in which the collapse of the lung is incomplete, except possibly in those in which the apex only is universally adherent. It is especially indicated for those cases with suspended apical cavities, particularly those which have increased in size after the artificial pneumothorax started because 
of adhesions which, dragging on the cavity wall, hold it rigidly open. In cases in which the base of the lung is adherent to the dome of the diaphragm, phrenicectomy is indicated either because the lung is held stretched out between the apex and the base, or because additional collapse of the lower lobe is needed on account of disease in that lobe.

Statistics shew that not only the immediate but also the late results, when phrenicetomy is combined with artificial pneumothorax, are very much better in all types of disease than when treatment is by pneumothorax alone.

On the voluntary or involuntary termination of artificial pneumothorax the lung, which has been long collapsed and has become progressively fibrosed and shrunken, has to re-expand in its endeavour once again to fill the hemithorax. Such a lung is incapable of re-expanding to its original volume. Drag occurs on the surrounding walls. The shifting of the mediastinum and the emphysema of the healthy lung parenchyma help to compensate, but do not abolish the strain of the intrapulmonary fibrous tissue within the diseased area and on the walls of the old cavities. To lessen this as much as possible, the dome of the diaphragm should be paralysed.

Permanent or temporary paralysis of the dome is also done in conjunction with, and usually as a preliminary to thoracoplasty and apicolysis. The writer is strongly of the opinion that the dome should not be permanently paralysed when a complete thoracoplasty is anticipated.

Phrenicectomy must be considered as an alternative to artificial pneumothorax in certain cases in which the disease is not extensive but for which some aid to sanatorium treatment is necessary or advisable. If it proves efficient the patient is saved the later serious interference with work of repeated refills and the risk of the complications of artificial pneumothorax. If the result is disappointing artificial pneumothorax can still be attempted.

Paralysis of the dome of the diaphragm is of very great value in the treatment of certain symptoms. The dry, spasmodic cough due to irritation of the diaphragm from the pull of an adherent lung can be completely stopped. The pain, ache or dragging sensation due to an old basal pleurisy can also be abolished. The writer makes it almost a rule to do a phrenicectomy in all cases of old pleurisy in which there is evidence of thickening of the basal pleura or of fixation of the diaphragm. This is not only for the treatment of symptoms but also, in cases with extensive sclerosis, as a prophylactic measure against the development later of bronchiectatic changes in the lung.

The writer has combined with phrenicectomy, the division of the scaleni muscles and sometimes the excision of the posterior end of the first rib. A review of the results in over 30 cases has, however, been found to be disappointing and he has therefore abandoned that operation.

\section{Thoracoplasty.}

The operation of thoracoplasty consists in the excision of lengths of bone from the posterior parts of the ribs: an essential being that the resection must extend right back to the transverse processes of the vertebræ. The excision may be of any number of ribs from the first 4 up to the first Io or II. Without excision of the first, an efficient collapse of the other ribs, all of which are slung up to the one above, is impracticable. The amount of each rib removed varies according to the extent and character of the disease. If the collapse is for the treatment of a case of chronic fibrotic disease with no obvious cavities, the extent of the 
resection is less than is necessary when there is a large rigid wall cavity. For such a cavity in the apex the total bone resection of the first 5 ribs may be as great as that for a ro-rib thoracoplasty in a non-cavity fibrotic case.

Indications-The operation of thoracoplasty does not come into consideration unless other less severe measures are impracticable or valueless. It is an operation essentially reserved for the chronic type of disease. It should not be done on patients with the exudative form of tuberculosis except in rare instances, such as when constantly recurring hæmorrhages, which cannot be checked by other means, are threatening the life of the patient. The risk of the operation in these cases is greatly increased, but is justified because the danger from the disease is so much greater.

Contraindications - Whilst the contraindications to artificial pneumothorax are few and elastic, those to a thoracoplasty are many and much more rigid. It is unwise to operate even on a chronic case if there has been a recent exacerbation, whether it is of the nature of an exudative spread or of an area of congestion round a cavity; such complications call, however, for temporary postponement only. The condition of the contralateral lung demands the greatest consideration. The presence of any active disease therein is usually an absolute contraindication: but there are exceptions. If the area affected is slight, is not recent and is not progressive and the character of the disease in the more affected lung is such that a thoracoplasty offers almost the only chance of saving life and of recovery, then the operation can be done, but with much greater caution as regards the number of ribs removed at any one stage. In these cases it is sometimes possible to reduce the risk from the contralateral lung by a preliminary course of gold therapy. The extent of old fibrotic changes in the better lung are also important as this lung will, if a complete thoracoplasty is done, be called upon to take on the whole of the work of blood aeration.

Whilst some surgeons are prepared to do a thoracoplasty in the presence of symptoms suggestive of intestinal tuberculosis, the writer maintains that the abdomen should be above suspicion. Laryngeal involvement is a contraindication in advanced cases when œdema or ulceration interferes with free air entry or efficient coughing. Provided there is no cardiac disease, a rapid, weak but regular pulse is not a bar to the operation, though much greater precautions and most careful premedication are necessary.

Premedication plays an important part in the success of the operation. Even in the most favourable cases there must be a preliminary period of absolute bed rest, cardiac tonics and the free ingestion of glucose. The immediate premedication consists of nembutal gr. 3 to $4 \frac{1}{2}$ in the preceding I2 hours, or alternatively paraldehyde, drachms 5 to 7 , in olive oil one hour before the regional anæsthetization. In both cases morphine gr. $\frac{1}{6}$ is given before the operation. More recently evipan is proving of great value as a substitute for this premedication.

The technique of the operation varies with the type and extent of the disease and with the amount of sputum. When there are not more than 25 c.c. of sputum in the 24 hours and it is controllable, that is to say the bronchial tubes can be emptied by coughing before the operation, no especial precautions are needed when giving the anæsthetic. With even smaller amounts of uncontrolled expectoration, or with larger amounts even though controlled, the head and shoulders of the patient should be lower than the buttocks during the operation and the anæsthetic should be given through an intratracheal tube so as to keep the passages. clear. 
A patient with generalized chronic fibrosis and no cavities, or only small ones and little or no sputum, can, if the general condition is satisfactory, be operated on in one stage with the resection of Io ribs. (Fig. 4, Plate I.) When such favourable conditions do not exist, the operation is done in two or even three stages at intervals of 2 to 3 weeks.

It has already been indicated that when large cavities exist a very much greater modification in the operation is necessary. A general relaxation of the lung is insufficient, the cavity walls must be relaxed and collapsed as well. To this end it is necessary to make a very extensive resection of all the overlying upper ribs. If a total thoracoplasty is indicated, the resection of the lower ribs need not be so great. When the disease is localized and the collapse of an apical cavity only is necessary there is always a possibility that the cavity may not collapse properly after a localized apical thoracoplasty, owing to the whole upper lobe and cavity being displaced downwards. In an endeavour to prevent this the extent of bone removed from the rib immediately below the cavity must be considerable. Should the cavity fail to be obliterated, a further resection of ribs at a second stage will be necessary. Incomplete collapse after a thoracoplasty can be supplemented by a paraffin filling, as mentioned later, or by.a further resection of ribs either through an axillary incision, or by the removal of the costal cartilages and anterior parts of the ribs through a parasternal incision.

\section{Apicolysis.}

Apicolysis is an operation which has recently, owing to improvements in technique, come again into favour. It fills in a gap in the range of possibilities of surgical endeavour. It makes possible the treatment of apical cavities when artificial pneumothorax and phrenicectomy have failed and thoracoplasty is contraindicated. The operation of apicolysis; or extrapleural paraffin " filling" is less severe than that of thoracoplasty. It can be done therefore as a means of obliterating cavities in patients over 45 years of age, at which age the average person is already too old for the major operation. It is justifiable also when there is active, but localised, disease in the contra-lateral lung. Paraffin "filling" can be used to supplement a thoracoplasty when a cavity has resisted complete collapse after the rib mobilization operation.

The route of access is either from behind, after resection of a length of bone from the $4^{\text {th }}$ rib, or from in front after resection of the second costal cartilage and adjacent piece of bone. The lung, together with the fused pleural membranes, is separated from the chest wall so as to free the entire apex and as much of the upper lobe as must be collapsed. The space thus formed is filled lightly with a special mixture of paraffin wax which is solid at body temperature.

Considerable difficulty may be experienced in separating the parietal pleura from the chest wall, owing to extrapleural adhesions. There is then a risk that the pleura. and lung will be torn and an opening made directly into the lung cavity, with probably serious results.

The smaller the cavity and the thinner the wall, the greater is the likelihood of a successful issue. Large cavities which require 300 c.c. to 400 c.c. of paraffin wax, or more, are difficult to obliterate and the risks of the operation are considerably increased. There is also a greater possibility that the "filling" will shift downwards towards the diaphragm, eventually producing a small localized collapse at the base while the cavity persists unaffected. 


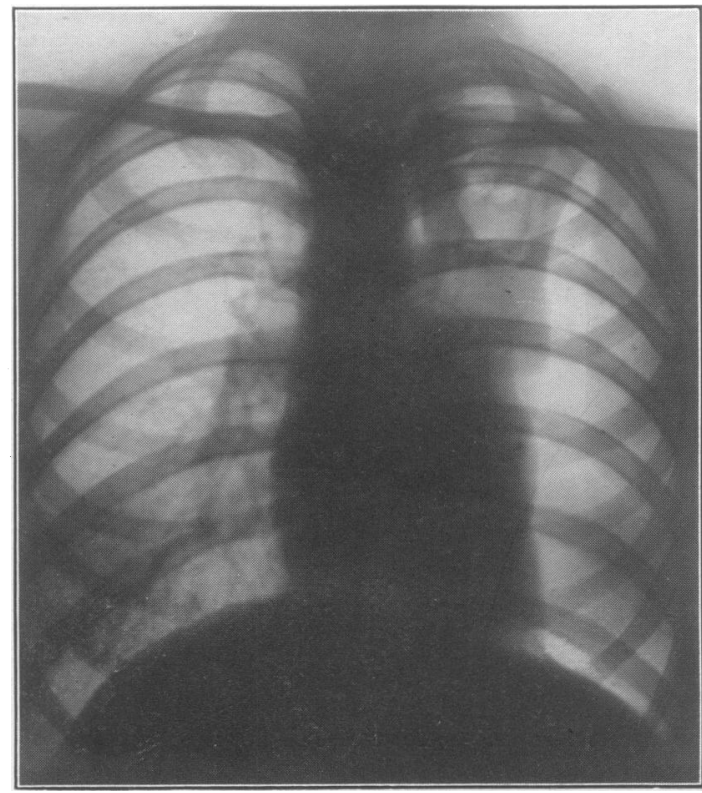

FIG. 1.

Artificial pneumothorax on left side, failing to control cavity in upper lobe owing to dense adhesion.

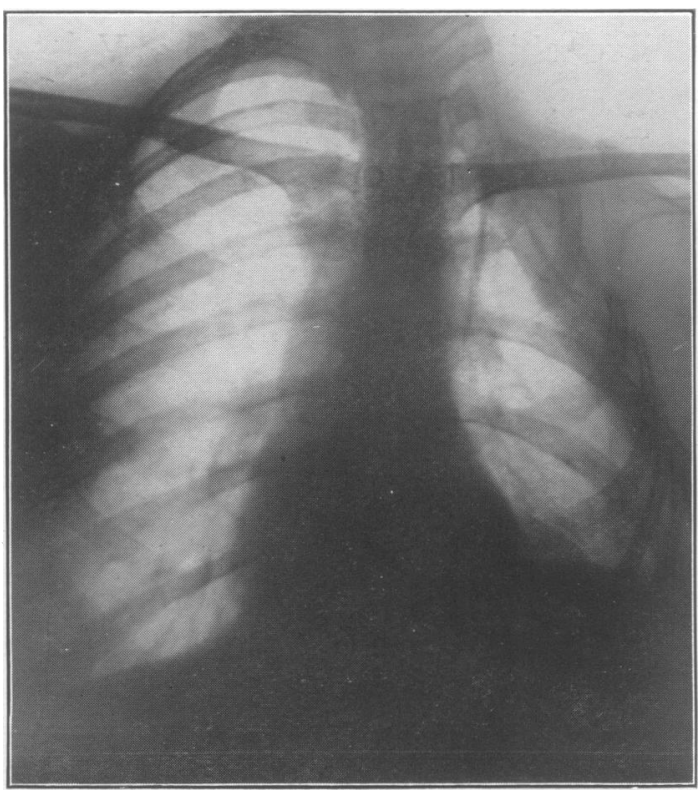

FIG. 3.

Apical 6-rib thoracoplasty.

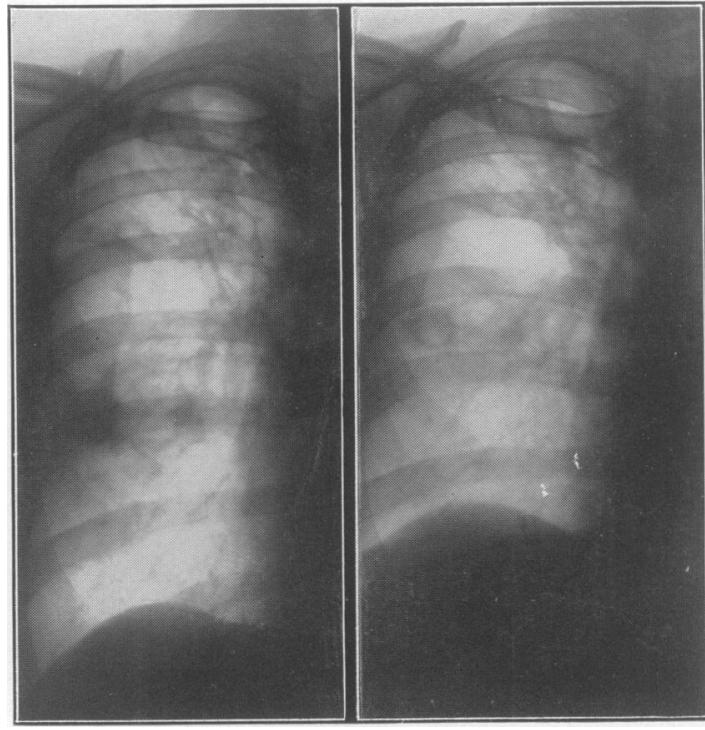

FIG, 2.

Shews the condition (left) before phrenicectomy, and (right) 3 months after the operation.

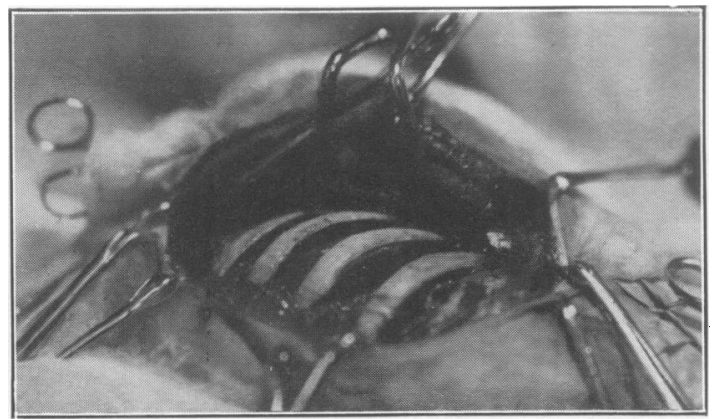

FIG. 4.

Exposure of ribs during thoracoplasty. 